\title{
Pre-Design of the Superconducting Magnet System for Magnum-psi
}

\author{
H. J. N. van Eck, W. R. Koppers, P. Smeets, A. den Ouden, W. J. Goedheer, N. J. Lopes Cardozo, and A. W. Kleyn
}

\begin{abstract}
The FOM-Institute for Plasma Physics Rijnhuizen is preparing the construction of Magnum-psi, a magnetized (3 T), steady-state, large area $\left(80 \mathrm{~cm}^{2}\right.$ ) high-flux (up to $10^{24} \mathrm{H}^{+}$ions $\mathrm{m}^{-2} \mathrm{~s}^{-1}$ ) plasma generator. The aim of the linear plasma device Magnum-psi is to provide a controlled, highly accessible laboratory experiment in which the interaction of a magnetized plasma with different surfaces can be studied in detail. Plasma parameters can be varied over a wide range, in particular covering the high-density, low-temperature conditions expected for the detached divertor plasma of ITER. A vital part of the Magnum-psi experiment is the superconducting magnet system, which generates a magnetic field of $3 \mathrm{~T}$ while good diagnostic access to the experiment is guaranteed.
\end{abstract}

In this contribution, we will explain the requirements on the magnet system, which is now in the pre-design phase. The present design consists of 3 cylindrical NbTi coils which generate a plateau shaped field of $3 \mathrm{~T}$ in a 1.3 meter room temperature bore. The discrete coils are supported by a $\mathbf{2 . 4}$ meter long single cylinder in a shared cryostat with 16 room temperature view-ports of $200 \mathrm{~mm}$ diameter. The field will most probably be passively shielded by an iron wall surrounding the experimental area.

As background, some elements of the pre-design of the Magnum-psi experiment; i.e. vacuum system, plasma source and diagnostics are presented.

Index Terms-Linear plasma generator, plasma simulator, plasma-surface interaction, superconducting magnets.

\section{INTRODUCTION}

$\mathbf{T}$ THE FOM Institute for Plasma Physics, in collaboration with its German (FZ-Juelich) and Belgian (ERM/KMS Brussels) partners in the framework of the Trilateral Euregio Cluster (TEC) agreement and the Eindhoven University of Technology, has launched a program to build a laboratory experiment in which the fundamental plasma-surface interaction (psi) processes can be studied in conditions similar to those in the ITER divertor. A large fraction of the effort in this program is now directed at the pre-design of Magnum-psi, the linear plasma generator which is capable of producing a long-pulse hydrogen/deuterium flux to a target of up to $10^{24} \mathrm{H}^{+}$ions $\mathrm{m}^{-2} \mathrm{~S}^{-1}$ at power fluxes of $10 \mathrm{MW} / \mathrm{m}^{2}$. The plasma beam has a diameter of $100 \mathrm{~mm}$ and the magnetic field

Manuscript received September 19, 2005. This work was supported by the European Communities under the Contract of Association between EURATOM/ FOM and by NWO and was carried out within the framework of the European Fusion Program. The views and opinions expressed herein do not necessarily reflect those of the European Commission.

H. J. N. van Eck, W. R. Koppers, P. Smeets, W. J. Goedheer, N. J. Lopes Cardozo, and A. W. Kleyn are with the FOM-Institute for Plasma Physics Rijnhuizen, Association EURATOM-FOM, Trilateral Euregio Cluster, the Netherlands (e-mail: vaneck@ rijnh.nl).

A. den Ouden is with the University of Twente, Faculty of Science and Technology, Low Temperature Division, Enschede, the Netherlands.

Digital Object Identifier 10.1109/TASC.2006.873271

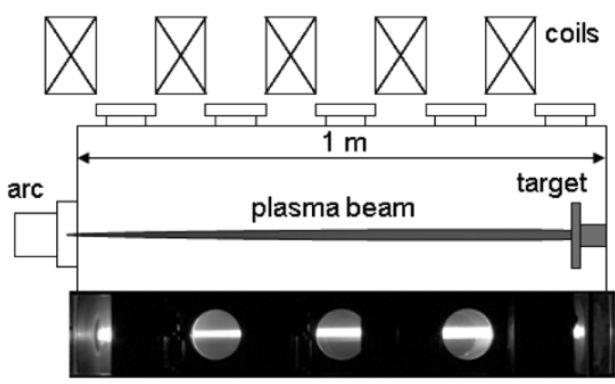

Fig. 1. A pure hydrogen plasma beam in a $0.4 \mathrm{~T}$ magnetic field as observed through the windows of the present Pilot experiment.

is variable up to $3 \mathrm{~T}$. A device providing these parameters is currently not available to the magnetic fusion community although a number of smaller devices do exist. These include the PISCES experiment at the University of California in San Diego [1], the PSI-2 experiment at the Humboldt University in Berlin [2] and NAGDIS-II at the Nagoya University [3].

The hydrogen plasma jet is produced with a wall stabilized DC cascaded arc [4] and expands supersonically into a vacuum vessel kept at low pressure. The axial magnetic field confines the expanding plasma jet to a narrow beam. In this way the plasma beam is transported to a target with minimal losses as can be seen in the already existing Pilot experiment [5] shown in Fig. 1.

In order to reach a sufficiently low pressure in the target region a three stage differential pumping scheme will be used. The target manipulator, additional plasma heating and the differential pumping impose a vacuum vessel diameter of $0.6 \mathrm{~m}$. This, together with space for diagnostics, pump ducts and wave guides, implies a magnet bore of $1.3 \mathrm{~m}$ diameter as can be seen in Fig. 2. Together with the required steady state operation and the minimization of the running costs the application of superconducting coils is imperative.

The pre-design of the magnet system has evolved [6] to a final stage, fully integrated with the Magnum-psi experiment.

\section{MAGNet SyStEM}

\section{A. Functional Design Requirements}

The magnetic field will be used to transport the plasma beam from the source to the target and to confine the particles that are ionized after coming of the target surface. The magnet system should therefore generate a plateau shaped magnetic field adjustable up to $3 \mathrm{~T}$ between the exit of the source and the end of the target plate. As can be seen in Fig. 3, the axial length of this region is $1750 \mathrm{~mm}$ while the width should be at least a $200 \mathrm{~mm}$ in diameter. The allowed field deviation on this field plateau 


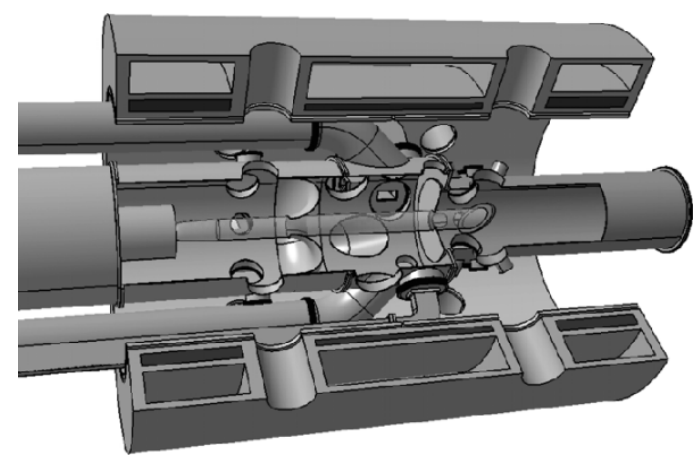

Fig. 2. Sketch of the Magnum-psi experiment. Shown are (from left to right) the source-, heating- and target chamber with pump ducts and wave guides inside the superconducting magnet. The plasma beam is guided through two skimmers by the magnetic field to the target plate.

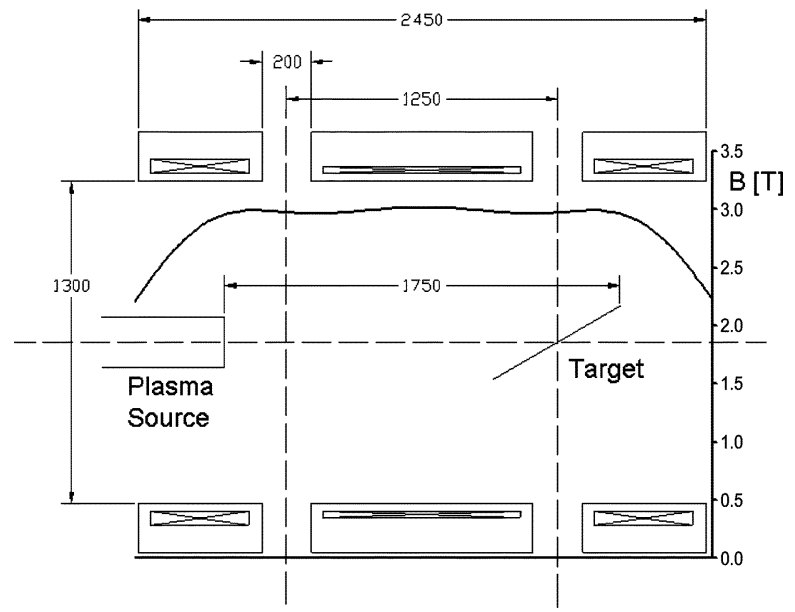

Fig. 3. Sketch of the magnet dimensions and magnetic field profile with respect to the plasma source and the target.

is $\pm 5 \%$. An important feature of the magnet system is the diagnostic access to the experiment which consists of 8 radial distributed access ports of $200 \mathrm{~mm}$ diameter each at two axial positions (source and target). These holes are required for laser diagnostics (e.g. Thomson scattering), fast retracting probes and sample holders. For the other diagnostics and control tools (e.g. Sum Frequency Generation) the room temperature bore of the magnet has to be freely accessible.

For maintenance on the experimental setup the complete magnet system will be placed on a rail system which allows the magnet to move over a distance of $2.5 \mathrm{~m}$.

\section{B. Principle Design of the Coils}

The basic magnetic design consists of 3 cylindrical NbTi superconducting coils operating at $4.3 \mathrm{~K}$. Apart from the main coils, shield coils can be envisaged at a larger radius carrying an opposite current to lower the stray field.

In the pre-design the use of a copper stabilized ( $\mathrm{Cu} / \mathrm{S}$ ratio: 4$)$, insulated NbTi composite superconductor with a cross section of $2.75 \mathrm{~mm}^{2}$ working at $4.3 \mathrm{~K}$ is assumed. The design current is $50 \%$ of its critical current, which corresponds to a $1.5 \mathrm{~K}$ temperature margin. The total thickness of the coil support, radiation shield, superinsulation and vacuum vessel is taken to be $50 \mathrm{~mm}$. Table I summarizes the main parameters for the actively and
TABLE I

PARAMETERS OF THE BASIC COIL DESIGN

\begin{tabular}{|c|c|c|c|}
\hline \multicolumn{4}{|l|}{ room temperature bore $[\mathrm{mm}]$} \\
\hline \multicolumn{3}{|c|}{ total length of the magnet system $L[\mathrm{~mm}]$} & 2450 \\
\hline \multicolumn{3}{|c|}{ number of coil sections $[\#]$} & 3 \\
\hline \multicolumn{3}{|l|}{ number of diagnostic ports [\#] } & 16 \\
\hline \multicolumn{3}{|c|}{ diameter of the radial ports [mm] } & 200 \\
\hline \multicolumn{3}{|c|}{ average field strength $[\mathrm{T}]$} & 3.0 \\
\hline \multirow{2}{*}{\multicolumn{3}{|c|}{$\begin{array}{l}\text { homogeneity of the field in the plateau region [\%] } \\
\text { estimated total weight }[\mathrm{kg}]\end{array}$}} & \pm 5 \\
\hline & & & 9000 \\
\hline & \multicolumn{2}{|c|}{ Active shielding } & Passive \\
\hline & Main coils & Shield coils & Main coils \\
\hline conductor length $[\mathrm{km}]$ & 133 & 85 & 74 \\
\hline operating current $[\mathrm{A}]$ & 375 & 375 & 442 \\
\hline current density $\mathrm{J}_{\mathrm{e}}[\mathrm{A} / \mathrm{mm} 2]$ & 136 & 136 & 161 \\
\hline max. field on conductor $[\mathrm{T}]$ & 6.0 & $<1$ & 5.3 \\
\hline induction $[\mathrm{H}]$ & 616 & 245 & 196 \\
\hline stored energy $[\mathrm{MJ}]$ & 43 & 17 & 19 \\
\hline force on most outer coil $[\mathrm{kN}]$ & -6630 & 2560 & -3480 \\
\hline
\end{tabular}

passively shielded design. Since the field homogeneity specification applies only to a region close to the magnet axis the incorporation of diagnostic holes does not result in a complex coil design. The resulting magnetic field profile can be seen in Fig. 3. Note that the maximum outer diameter of the magnet is limited to $2400 \mathrm{~mm}$.

\section{Cryostat}

Characteristic for both the active and passive shielded systems are the large axial forces exerted by the most outer coils. A reliable support system to sustain these forces is a crucial element for successful operation of the system at $3 \mathrm{~T}$. In the pre-design all main coils are wound on one cylindrical coil former equipped with holes to accommodate the radial ports. The coil former is surrounded by both a radiation shield and vacuum vessel at the inner and outer diameter. Additional space is required at the outer diameter to accommodate other cryogenic components. The $300 \mathrm{~K}$ radial ports each contain a vacuum wall, a radiation shield and superinsulation which are piecewise connected to the corresponding parts at the inner and outer diameter. Fig. 4 gives an impression of the complete system.

Another approach is to put the discrete coils into separate cryostats. The space between the cryostats can be used for diagnostic access. Due to the large axial forces between the coils these modules have to be connected by a series of cold support rods, each surrounded by a connective radiation shield and a connective vacuum vessel. This makes the cryogenic design rather complex and expensive, but not impossible. However, further study is required.

\section{Magnetic Shielding}

The magnetic stray field should be shielded down to the 5 Gauss level outside the experimental area which is defined at 14.8 by $8.4 \mathrm{~m}$ surrounding the magnet. Without shielding the 5 Gauss line lies approximately 15 meters away from the magnet. From the parameters in Table I it is evident that the use of active shielding makes the design more complex. The amount of superconductor is greatly enlarged as well as the 


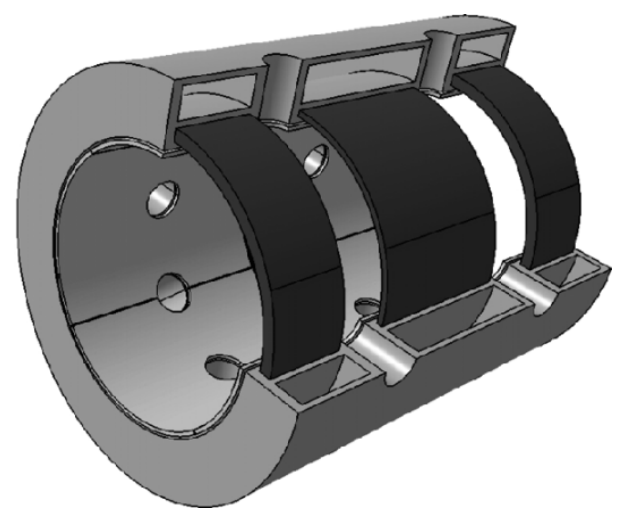

Fig. 4. Sketch of the magnet system including the water cooled heat shield.

induction and the stored energy. From both technological and economical point of view passive shielding is the preferred solution for this magnet system. Since passive shielding directly onto the magnet cryostat would greatly limit the accessibility, an iron dome surrounding the whole magnet is foreseen. By placing $100 \mathrm{~mm}$ thick iron walls around the experimental area effective shielding is accomplished. However, to make the demands for the iron wall less stringent and to lower the weight on the floor, a hybrid solution, being active shielding near the magnet in combination with a thinner iron wall might be desirable. The cost of the iron shield plus civil engineering to support the weight will have to be balanced against the cost of active shielding.

\section{E. Heat Load and Cooling}

The design of the cryogenic cooling system is mainly driven by demands concerning minimization of running costs and the mode of operation. A purely conduction cooled system would limit the ramp time due to hysteresis losses in the conductor and eddy current losses in the system. In order to keep ramp-up and ramp-down times to an acceptable level it is concluded that a recondensing helium system cooled by cryocoolers is favorable.

There are two ways of realizing a recondensing helium system. In the first system the coils are fully emerged in liquid helium which is cooled by coolers placed onto the service neck of the cryostat. The evaporated helium recondenses at the cold finger of the cryocooler and is fed back passively to the coils. The second system has the coils in vacuum and uses thermosiphons from a small helium bath to cool the coils. The latter arrangement might be more cost effective since there is no need for a large helium vessel. Further investigation is ongoing.

During a plasma pulse and during vacuum wall conditioning the plasma chamber and pump ducts are expected to reach a temperature of $150{ }^{\circ} \mathrm{C}$. The magnet is therefore shielded from the heat radiated from the experiment by an extra water cooled heat shield.

\section{MAGNUM-PSI PRE-DeSIGN}

\section{A. Vacuum System}

In Magnum-psi a comparable ion to neutral ratio near the target as in the ITER divertor must be reached. Therefore the

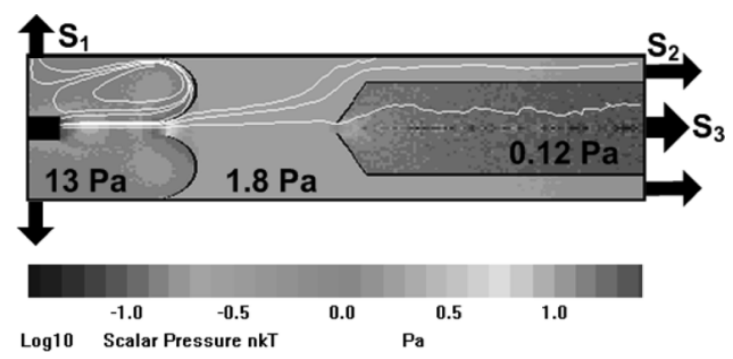

Fig. 5. 2D DSMC simulation of $75 \mathrm{slm}$ hydrogen gas expanding in a differentially pumped vacuum system. In this case the pumping speeds $\mathrm{S}_{1}=\mathrm{S}_{2}=$ $\mathrm{S}_{3}=38.000 \mathrm{~m}^{3} / \mathrm{h}$. Some flow lines are shown for clarity.

Magnum vacuum system must be able to reach the required neutral pressure while the plasma source puts in 75 std. liter per min. (slm) of gas, which is only partly ionized $(\sim 10 \%)$. Neutrals are pumped away while the ions can reach the target due to the magnetic field. To pump away the high gas load and obtain low enough pressure at the target area a 3 stage differential pumping solution is introduced. In Fig. 5 the result of a Monte Carlo simulation for neutral hydrogen gas is shown. This simulation is performed using the DS2V program by G. A. Bird [7]. The hydrogen gas expands supersonically into the source chamber (first stage) where a large part of the gas load is reversed by the first skimmer and pumped away by two large mechanical roots pumps. The heating chamber (second stage) is also pumped by a set of roots pumps due to the high gas load. This simulation shows that the neutral pressure in the target chamber (third stage) is low enough to ensure experimental conditions similar to those found in the ITER divertor. Hydrogen plasma produced in the plasma source follows the magnetic field lines, crosses the two skimmers and recombines at the target surface. The resulting pressure near the target is therefore expected to increase up to $1 \mathrm{~Pa}$. The target chamber will be pumped by an array of external cryopumps cooled by mechanical cryocoolers. This pumping option requires no liquid cryogens, has a variable pumping speed and a high capacity which will ensure an extensive measurement time.

\section{B. Plasma Source and Heating}

One of the key goals for Magnum-psi is to achieve plasma conditions at the target close to those expected for ITER. The ITER divertor plates sit at a grazing incidence angle with respect to the plasma which reduces the surface heat and particle fluxes down to the $10 \mathrm{MWm}^{-2}$ and $10^{24} \mathrm{~m}^{-2} \mathrm{~s}^{-1}$ level. The plasma heating power in Magnum-psi will be provided by 3 distinct systems; the cascaded arc plasma source, Ohmic heating and RF heating. Modeling and experiments are ongoing.

\section{Diagnostics}

A large number of plasma and surface diagnostics will be installed on Magnum-psi. To guarantee reproducible experiments the exact plasma parameters (e.g. density and temperature) in front of the target will have to be known. Most important plasma diagnostics include Thomson scattering and high resolution optical emission spectroscopy.

It will be important to be able to monitor some of the surface properties during operation (in-situ) to have a means of 
control. Therefore a spectroscopic measurement based on vibrational Sum Frequency Generation (SFG) [8] is implemented. This technique is expected to be compatible with the harsh conditions in Magnum-psi.

To further determine the properties of the surface that are modified in the interaction with the plasma a range of ex-situ diagnostics will be installed, including X-ray photoelectron spectroscopy (XPS), erosion and deposition monitors (EDM), Secondary Ion Mass Spectroscopy (SIMS) and Thermal Desorption Spectroscopy (TDS).

\section{CONCLUSION}

- The $3 \mathrm{~T}$ plateau shaped magnetic field is generated by a single layer coil system consisting of 3 cylindrical coils.

- 16 radial ports and the large room temperature bore guarantee good diagnostic access to the experiment.

- Effective shielding is accomplished by $100 \mathrm{~mm}$ thick iron walls surrounding the experimental area.

- A recondensing helium system cooled by cryocoolers is operational easy and economic.

- The pre-design of Magnum-psi is reaching its final stage. The design and manufacturing phase will commence shortly. First measurements are expected in 2009.

\section{ACKNOWLEDGMENT}

The authors would like to thank J. Huinck for providing the illustrations of the pre-design (Figs. 2 and 4).

\section{REFERENCES}

[1] E. M. Hollmann, A. Y. Pigarov, R. Seraydarian, D. G. Whyte, and S. I. Krasheninnikov, "Particle balance measurements during detachment in a gas-target diverter simulator," Phys. Plasmas, vol. 9, no. 4, pp. 1226-1232, Apr. 2002.

[2] H. Grote, W. Bohmeyer, H. Reiner, H. Behrendt, G. Fussmann, H. Meyer, D. Naujoks, and E. Pasch, "Comparison of chemical sputtering yields for different graphites at high ion flux densities," J. Nucl. Mater., vol. 241-243, pp. 1152-1155, 1997.

[3] E. M. Hollmann, D. G. Whyte, D. Nishijima, N. Ohno, Y. Uesugi, and N. Ezumi, "Evidence for the importance of radial transport in plasma detachment in the Nagoya University Divertor Simulator (NAGDISII)," Phys. Plasmas, vol. 8, no. 7, pp. 3314-3320, Jul. 2001.

[4] M. C. M. van de Sanden, G. M. Janssen, J. M. de Regt, D. C. Schram, J. A. M. van der Mullen, and B. van der Sijde, "A combined ThomsonRayleigh scattering diagnostic using an intensified photodiode array," Rev. Sci. Instrum., vol. 63, no. 6, pp. 3369-3377, Jun. 1992.

[5] B. de Groot, Z. Ahmad, R. P. Dadiya, R. Engeln, W. J. Goedheer, N. J. Lopes Cardozo, and V. Veremiyenko, "Magnum-psi, a new linear plasma generator for plasma-surface interaction studies in ITER relevant conditions," Fusion Eng. Des., vol. 66-68, pp. 413-417, 2003.

[6] H. J. N. van Eck, A. den Ouden, G. J. van Rooij, W. J. Goedheer, B. de Groot, N. J. Lopes Cardozo, and A. W. Kleyn, "A 3 T magnet system for Magnum-psi," IEEE Trans. Appl. Supercond., vol. 15, no. 2, pp. 1303-1306, Jun. 2005.

[7] G. A. Bird, Molecular Gas Dynamics and the Direct Simulation of Gas Flows. New York: Oxford University Press Inc, 1994.

[8] S. Roke, "New Light on Hidden Surfaces," Ph.D. thesis, Leiden University, The Netherlands, 2004. 